\title{
La protéine p53, une sonde pour explorer la nature des facteurs oncogéniques dans l'environnement
}

La protéine p53 est un anti-oncogène qui, lorsqu'il est modifié, acquiert des propriétés oncogéniques $\left(\mathrm{m} / \mathrm{s} \quad n^{\circ} 8\right.$, vol. 5, p. 598 et $n^{\circ} 8$, vol. 6, p. 821). Cet anti-oncogène est, de loin, celui qui est le plus fréquemment modifié dans les tumeurs humaines (dans environ $55 \%$ des observations), si bien que l'on peut se demander si son altération n'est pas, en fait, un pré-requis au développement tumoral. Contrairement aux oncogènes de la famille p21 ras, dont l'activation exige la mutation très précise de certains codons (par exemple les codons 12 et 61), l'inactivation du pouvoir antioncogénique de p53 peut être la conséquence d'une grande diversité d'altérations de la protéine. En d'autres termes, un très grand nombre de mutations ou d'autres modifications de p53 ont été, d'ores et déjà, caractérisées dans différentes tumeurs humaines. C'est dire l'intérêt de certaines observations dans lesquelles une maladie particulière, dans un environnement géographique donné, est associé à une mutation spécifique de p53. Ainsi Ozturk et al. (Charleston, MA, USA) [1] ont-ils démontré la fréquence d'une transversion $\mathrm{G} \rightarrow \mathrm{T}$ transformant l'arginine 249 en sérine dans les hépatomes observés en Afrique et en Asie du Sud-Est. Une telle mutation est exceptionnelle dans d'autres tumeurs observées en d'autres régions. Les facteurs de risques expliquant le caractère endémique de l'hépatocarcinome dans les régions étudiées par les auteurs sont l'infection par le virus de l'hépatite $\mathrm{B}$ (VHB) et l'aflatoxine $\mathrm{B} 1$. Le pouvoir mutagène de ce dernier agent comporte la formation de résidus désoxyguanosine entraînant un remplacement d'un $G$ par un T... C'est-à-dire exactement $\mathrm{m} / \mathrm{s} n^{\circ} 3$, vol. 8, mars 92 le type d'altération notée par Ozturk et ses collègues. La responsabilité de l'aflatoxine B1 est d'ailleurs confirmée par l'observation que des carcinomes hépatocellulaires d'autres régions, non soumis à l'action de l'aflatoxine alimentaire mais très souvent reliés à une infection chronique par le VHB, ne sont pas associés à cette mutation de l'arginine 249.

Dans les cancers du poumon, l'agent étiologique principal, voire quasi exclusif, est, en toutes régions, la fumée de cigarette... dans laquelle le benzo(a)pyrène est le carcinogène majeur. Ce dernier agit un peu comme l'aflatoxine B1, modifiant des $\mathrm{G}$ et entraînant, par conséquent, des transversions G/C $\rightarrow$ T/A. De fait, de telles mutations représentent $46 \%$ des anomalies détectées au niveau de p53 dans ce cancer [3], mais sont ici réparties en de nombreux codons. En dehors de l'action de mutagènes particuliers, les mutations spontanées les plus fréquentes sont des transitions $\mathrm{C} / \mathrm{G} \rightarrow$ T/A et $\mathrm{G} / \mathrm{C} \rightarrow$ AT survenant au niveau de dinucléotides $\mathrm{CpG}$. De fait, ceux-ci sont généralement méthylés chez les vertébrés et la méthylcytosine subit une désamination en thymine 3,5 fois plus rapide que la cytosine non méthylée $[4,5]$. Quoique, du fait de mutations récurrentes au cours de l'évolution, les dinucléotides $\mathrm{CpG}$ soient très sous-représentés chez les vertébrés, ils sont encore à l'origine de 30 à $40 \%$ des mutations de la lignée germinale chez l'homme mais ne représentent que moins de $10 \%$ des mutations de p53 dans le cancer du poumon [5]. En revanche, les transitions $\mathrm{G} / \mathrm{C} \rightarrow \mathrm{A} / \mathrm{T}$ au niveau de dinucléotides $\mathrm{CpG}$ forment $76 \%$ des mutations de p53 dans le cancer du côlon, avec deux points chauds majoritaire- ment mutés au niveau des codons 175 et 273 [5]. Cela peut représenter la répartition des mutations " endogènes " de p53, suggérant que, dans le cancer du colon, cette protéine n'est pas altérée du fait de l'action d'un carcinogène spécifique. Ces mutations " naturelles ", peutêtre stimulées par des événements antérieurs de la progression tumorale [inactivation des anti-oncogènes APC $\left(\mathrm{m} / \mathrm{s} n^{\circ} 7\right.$, vol. 7, p. 718), DCC $(\mathrm{m} / \mathrm{s}$ $n^{\circ}$ 3, vol. 6, p. 292), activation de p21 ${ }^{\text {ras }}$ et, du fait de l'avantage prolifératif ainsi conféré, ne seraient que sélectionnées par leur aptitude à provoquer une croissance cellulaire encore accélérée.

Brash et al. (New Haven, CT ; Boston, MA ; New York, NY, USA et Uppsala, Suède [2]) ont, quant à eux, rapporté la présence de mutations $\mathrm{C} \rightarrow \mathrm{T}$ de $\mathrm{p} 53$ dans $58 \%$ des épithéliomas cutanés spinocellulaires, au niveau de dimères de pyrimidine. Or, il est bien connu que les rayons ultraviolets, agents étiologiques en cause dans la majorité des cancers cutanés, agissent spécifiquement sur de tels dimères de pyrimidine. Particulièrement caractéristiques de l'action des UV sont les doubles changements de bases CC $\rightarrow$ TT... observés dans trois cas de tumeurs de la peau.

Ces résultats sont extraordinairement importants, pour des raisons théoriques aussi bien qu'épidémiologiques. Ils démontrent en effet que p53 peut être l'un des agents expliquant la spécificité des carcinogènes potentiels, physiques ou chimiques. Cette spécificité suggère également que l'altération de p53 joue un rôle tout à fait essentiel dans le déclenchement de la carcinogenèse. Si l'inactivation de p53 était, en effet, un phénomène (n) 
relativement tardif, secondaire à de premières étapes de progression tumorale, on peut supposer que le type d'altérations observées dépendrait moins du facteur oncogénique initial et refléterait plus les mécanismes endogènes de mutation, comme cela est observé pour le cancer colique. Au point de vue épidémiologique, ces exemples suggèrent qu'il pourrait également être possible d'établir une corrélation entre la carcinogenèse due à certains toxiques (tabac, alcool, dérivés aromatiques et benzéniques...) et des mutations très particulières de p53 qui pourrait être ainsi un outil particulièrement précieux pour de telles investigations. Enfin, il faut noter que les résultats de Ozturk et Brash attirent l'attention sur deux carcinogènes naturels, c'est-à-dire qui ne sont pas des conséquences du développement de l'industrialisation, ce qui est une pièce à verser au dossier des discussions actuelles sur les risques réels des différents carcinogènes présomptifs $[6$, 7].

A. $\mathbf{K}$.

1. Ozturk M, et al. p53 mutation in hepatocellular carcinoma after aflatoxine exposure. Lancet 1991 ; 338 : 1356-9.

2. Brash DE, Rudolph JA, Simon JA, et al. A role for sunlight in skin cancer: UV-induced p53 mutations in squamous cell carcinoma. Proc Natl Acad Sci USA 1991 ; 88 : 10124-8.

3. Puisieux A, Lims S, Groopman J, Ozturk M. Selective targeting of p53 gene mutational hot spots in human cancers by etiologically defined carcinogens. Cance Res 1991 ; 51 : 6185-9.

4. Jordan BB. Ilôts HTF : le gène annoncé. médecine/sciences $1991 ; 7: 153$.

5. Jones PA, Rideout WM, Shen JC, Spruck $\mathrm{CH}$, Tsai YC. Methylation, mutation and cancer. Bioessays 1992 ; 14 : 33-6.

6. Vogelstein P, Kinzler KW. Carcinogens leave fingerprints. Nature 1992 ; 355 : 209-10.

7. Tubiana M. L'épidémiologie du cancer. méde-

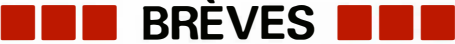

Le PNA : un analogue structural de l'ADN aux propriétés étonnantes. Les réactifs et médicaments dérivés d'oligonucléotides sont d'un grand intérêt potentiel : on peut espérer modifier l'expression d'un gène cible, soit par interaction au niveau de l'ARNm grâce à des oligonucléotides anti-sens, soit directement au niveau du gène grâce à la formation d'une triple hélice. Dans ce cas, l'interaction se fait par l'intermédiaire de liaisons de Hoogsteen entre les bases d'un oligonucléotide et les bases d'un des brins de l'ADN selon un mode d'appariement spécifique, mais qui n'existe que pour les pyrimidines (interactions T-A/T et C-G/C), ce qui limite le choix des séquences cibles. Cependant, in vivo, les oligonucléotides sont rapidement dégradés et traversent difficilement les membranes cellulaires. Pour pallier ces inconvénients, des modifications de la chaîne phosphodésoxyribosique (thiophosphates, méthylphosphates, etc.) ont été utilisées, mais elles sont techniquement difficiles et la stabilité des liaisons avec les acides nucléiques est souvent diminuée. Une approche plus radicale a été entreprise par une équipe danoise [1] qui rapporte la synthèse d'un ersatz d'ADN, dans lequel l'ensemble de la chaîne phosphodésoxyribosique a été remplacée par une chaîne polyamide (poly 2-aminoéthylglycine) sur laquelle ont été greffées des bases normales, placées à bonne distance par des groupes méthylène-carbonyl. La molécule PNA (polyamide nucleic acid) porte dix résidus thyminyl, et, en outre, possède à l'une de ses extrémités un résidu lysine destiné à augmenter son hydrophobicité et à stabiliser son interaction avec des acides nucléiques. L'autre extrémité peut porter un intercalant dérivé de l'acridine. Dans une première expérience, les auteurs qui étudiaient la possibilité de liaison de PNA avec son brin complémentaire $\mathrm{ADN}$ (A10) eurent la surprise de constater que non seulement l'hybridation PAN-A10 était possible, mais que, de plus, elle restait stable dans des conditions dénaturantes (urée 7M). Le Tm (température pour laquelle $50 \%$ des hybrides sont fon- dus) en présence de $50 \%$ de formamide passe de $23^{\circ} \mathrm{C}$ pour le double brin naturel $\mathrm{A} 10-\mathrm{T} 10$ à $73^{\circ} \mathrm{C}$ pour l'hybride PAN-A10, et même à $83^{\circ} \mathrm{C}$ quand la molécule possède un groupement acridine. Dans une deuxième série d'expériences, les auteurs ont étudié l'interaction de la molécule simple brin PAN avec la séquence double brin A10-T10 insérée dans un fragment d'ADN plasmidique. Les auteurs s'attendaient à la formation d'une triple hélice dans laquelle PAN aurait interagi avec $\mathrm{A} 10$, mais la réactivité de celui-ci vis-à-vis de plusieurs réactifs était peu modifiée. En revanche, le brin $\mathrm{T} 10$, supposé ne pas être modifié, devenait fortement sensible à la nucléase $\mathrm{S} 1$ et au permanganate de potassium, agents réagissant préférentiellement avec l'ADN simple brin. Tout indiquait donc que le PAN avait déplacé la séquence T10 pour s'apparier avec A10, par des liaisons classiques de Watson-Crick, le brin complémentaire T10 restant non apparié. Cette immixtion de PAN au sein de l'ADN double brin se produit, dans les conditions expérimentales utilisées, en 20 minutes environ, probablement à l'occasion d'une "respiration" de l'ADN (la dissociation transitoire et brève des deux brins). Ce mode d'interaction entre PAN et l'ADN permet d'espérer que des séquences non polypyrimidiques pourront, elles aussi, être utilisées, augmentant ainsi le nombre des cibles potentielles. Si la très haute stabilité des hybrides ne diminue pas la spécificité de l'interaction, d'immenses espoirs seront permis quant à la possibilité de cibler directement des gènes au sein d'une cellule, en particulier pour le traitement d'anomalies génétiques somatiques (mutation de proto-oncogènes, intégration de provirus...).

[1. Nielsen P, et al. Science $1991 ; 254$ 1497.] 
DI Lésions moléculaires dans le diabète insipide familial. Le diabète insipide (DI), dont les symptômes principaux sont la soif et la polyurie, est dû à l'insuffisance du nonapeptide AVP (arginine vasopressine) qui régit la réabsorption rénale de l'eau et est sécrété dans l'hypothalamus. L'AVP est synthétisé à partir du précurseur AVP-NP, incluant la neurophysine et une glycoprotéine C-terminale. Le gène siège sur le chromosome 20 , au voisinage de celui de l'ocytocine. Il est divisé en 3 exons, l'AVP étant codé par le premier. On pourrait concevoir la production du DI par une anomalie du nonapeptide lui-même, mais aussi par atteinte d'une autre région du gène, empêchant par exemple le précurseur d'atteindre son lieu de destination, les vésicules sécrétoires, ou de subir sa maturation normale. C'est ainsi qu'on interprète le modèle animal que constitue le rat Brattleboro, à transmission récessive autosomique : c'est une délétion d'une base dans le $2^{e}$ exon, qui code pour la neurophysine, qui provoque la formation d'une protéine dont l'extrémité C-terminale est anormale [1]. Chez l'homme, le DI est le plus souvent sporadique, "idiopathique ", mais il en existe de rares formes familiales, dont l'hérédité est dominante. Deux équipes, l'une de Nagoya (Japon), l'autre de Hambourg (RFA) et d'Amsterdam (PB), ont entrepris l'exploration de ces formes. Chez deux sujets d'une famille, Ito et al. [2] ont trouvé une mutation ponctuelle du codon 57, Gly $\rightarrow$ Ser, dans l'exon 2 codant pour la neurophysine ; Bahnsen et al. [3], dans une famille néerlandaise, ont observé chez tous les membres atteints une mutation Gly $\rightarrow$ Val, qu'ils situaient en position 17 de la neurophysine (qui représente $70 \%$ du précurseur), ce qui correspond au codon 29, de l'ensemble. Ces mutations étaient à l'état hétérozygote. Dans les deux cas, elles altèrent des sites d'enzymes de restriction pour des enzymes appropriées, ce qui rend le criblage facile ; mais cette méthode a l'inconvénient de laisser passer toute anomalie autre que celle que l'on cherche. Il reste ainsi des familles, françaises et américaines [4], qui n'ont été explorées qu'incomplètement. Phénomène remarquable, la vingtaine de cas non familiaux examinés n'a montré aucune anomalie, et l'origine des troubles reste à déterminer dans le DI idiopathique.

On a déjà pu remarquer que les DI familiaux sont génétiquement dominants alors que la forme du rat est récessive. Fait apparemment paradoxal, c'est la gravité plus grande de la lésion moléculaire du rat qui rend la maladie plus bénigne. Chez le rat, la mutation décalage aboutit à modifier complètement la fin de la protéine ; celleci reste séquestrée dans le système réticulo-endothélial, et le produit du gène normal peut remplir son office chez l'hétérozygote. Chez l'homme, les mutations ponctuelles n'empêchent pas le transport du précurseur au moins dans ses premiers stades. Il y a probablement interférence du produit altéré avec le normal au cours du transport ou de l'interaction avec les récepteurs. Des études sur cultures de cellules après transfection du gène normal ou muté aideront probablement à comprendre les mécanismes en cause. [1. Schmale $\mathrm{H}$, et al. Eur J Biochem $1989 ; 182: 621-7$.

[2. Ito M, et al. J Clin Invest 1991 ; 87 : 725-8.]

[3. Bahnsen U, et al. EMBO J 1992 ; 11: 19-29.]

[4. Repaske DR, et al. J Clin Endocr Metab 1990 ; 70 : 752-7.]

- Un homologue du facteur de ranscription NF- $\kappa$ B modifié dans des leucémies humaines. La plupart des leucémies comportent des remaniements chromosomiques dont il a été montré qu'ils aboutissaient très fréquemment à l'activation d'oncogènes ( $m / s n^{\circ} 5$, vol. 6, p. $489 ; n^{\circ} 8$, vol. 7, p. 880). Par ailleurs, les connaissances que nous avons, à l'heure actuelle, de l'oncogenèse font de n'importe quel gène codant pour un activateur potentiel de la croissance cellulaire, un possible oncogène. Ainsi, les protéines de la famille NF- $\kappa \mathrm{B}$, l'un des intermédiaires de l'action mitogénique de la protéine kinase $\mathrm{C}$, sont-elles dans ce cas ( $\mathrm{m} / \mathrm{s} n^{\circ} 1$, vol. 7, p. 67). On connaît maintenant plusieurs membres de cette famille capables, sous forme de dimères ou de tétramères, de reconnaître le même type d'élément d'ADN grâce à un domaine protéique équivalent à celui des oncogènes de type rel. On connaît ainsi p50 NF- $\kappa \mathrm{B}, \mathrm{p} 65 \mathrm{NF}-\kappa \mathrm{B}$, $c-R e l$ et son équivalent viral, v-Rel. Une équipe américano-italienne de New York et Milan vient d'isoler un nouveau membre de cette famille remanié par une translocation chromosomique $\mathrm{p}(10 ; 14)(\mathrm{q} 24 ; \mathrm{q} 32)[1]$ chez les malades atteints de lymphome non hodgkinien développé aux dépens des cellules B lymphocytaires. Ce nouveau gène est appelé lyt-10 ; il est transloqué à proximité du fragment $\mathrm{C} \boldsymbol{\alpha}$ des gènes de chaîne lourde d'immunoglobulines, sur le chromosome 14. De plus, le remaniement chromosomique entraîne une troncation du gène lyt-10 dans la région codant pour la partie carboxy-terminale de la protéine. Le produit de $l y t-10$ est très similaire au précurseur de $105 \mathrm{kDA}$ de p50 NF- $\kappa \mathrm{B}$ ( $m / s n^{\circ} 1$, vol. 7, p. 67). Dans ce dernier, la partie amino-terminale code pour p50 alors que la partie carboxyterminale, contenant des répétitions de type ankyrine, joue un rôle inhibiteur sur la translocation de p50 dans le noyau et sur la fixation à l'ADN. La troncation de cette région dans lyt-10 peut donc activer le produit correspondant. Ce gène modifié se trouve, par ailleurs, dans une zone de chromatine active dans les cellules lymphocytaires $B$, ce qui peut expliquer son hyperexpression dans ces cellules. C'est cette activation par troncation carboxyterminale et hyper-expression qui pourrait expliquer le rôle oncogénique probable du produit du gène lyt-10.

[1. Neri A, et al. Cell 1991; 67 : 1075-87.]

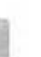

$m / s n^{\circ} 3$, wol. 8 , mars 92 
Présence d'une activité aldostérone synthétase dans la zone fasciculée de la corticosurrénale : une cause rare d'hypertension artérielle familiale. Les hyperaldostéronismes primaires sont soit l'expression d'une tumeur issue de la zone glomérulée de la corticosurrénale (adénome de Conn), soit celle d'une hyperplasie bilatérale des surrénales. Beaucoup plus rares sont les hyperminéralocorticismes secondaires à un déficit enzymatique touchant la $17 \alpha$ hydroxylase ou la $11 \beta$-hydroxylase. Dans ces deux dernières affections, l'absence de cortisol est responsable d'une hyperplasie congénitale des surrénales avec, dans le premier cas, un excès de production de désoxycorticostérone (DOC) et d'aldostérone et, dans le deuxième cas, un excès de production de DOC et d'androgènes surrénaliens. La présence anormale d'une activité aldostérone synthétase dans la région fasciculée est une autre cause rarissime d'hyperminéralocorticisme*. Cette forme familiale d'hypertension artérielle, observée dès la petite enfance, sans anomalie du développement gonadique ni virilisation précoce, et dont la transmission est autosomique dominante, se caractérise par des taux sériques élevés de 18-oxocortisol et de 18-hydroxycortisol, métabolites du cortisol normalement non détectables. Ces métabolites circulants témoignent d'une activité enzymatique 18-hydroxylase (normalement exclusivement présente dans la zone glomérulée) dans la zone fasciculée. Selon une étude récente [1], cette anomalie d'expression serait la conséquence d'un crossing over inégal plaçant sur l'un des chromosomes 8 de tous les membres atteints d'une même fratrie, la partie codante du gène de la 18-hydroxylase sous contrôle des régions régulatrices du gène de la $11 \beta$-hydroxylase, dont l'expression, limitée aux zones fasciculée et réticulée, est réprimée par l'ACTH.

\footnotetext{
- Rappelons que l'aldosttrone est le produit final d'une oxydation du groupement méthyl en position 18 (18-hydraxylase) de la corticostérone, suivie d'une élape de réduction de la fonction alcool en aldéhyde (aldosterone)
}

Ce crossing over serait responsable du profil de restriction particulier et commun à tous les sujets atteints de cette famille. Cet accident méiotique serait favorisé par la forte homologie des séquences codantes (95\%) des gènes de ces deux enzymes dépendantes du cytochrome P450, et par leur proximité sur le chromosome 8 . Il rendrait compte non seulement de l'expression ectopique de l'enzyme dans la zone fasciculée, mais aussi de l'effet bénéfique des glucocorticoïdes dans cette affection : en freinant la sécrétion d'ACTH, ceux-ci inhiberaient indirectement l'expression du gène chimérique dans la zone fasciculée, alors que l'allèle non recombinant et exprimé dans la zone glomérulée resterait sous le contrôle normal du système rénine-angiotensine. Cette mutation n'est pas sans rappeler le déficit en $11 \beta$-hydroxylase, responsable d'une hyperplasie congénitale des surrénales, et conséquence, lui aussi, d'un crossing over inégal $\left(\mathrm{m} / \mathrm{s} n^{\circ} 9\right.$, vol. 2, p. 527).

[1. Lifton RP, et al. Nature 1992 ; 355 : 262-5.]

Le signal d'internalisation par endocytose des protéines membranaires. Il existe une famille de récepteurs membranaires qui, après contact avec leur ligand, sont internalisés par endocytose dans des puits, et ensuite dans des vésicules recouverts (coated pits and vesicles) [1]. D'autres protéines, qui ne sont pas des récepteurs, subissent de semblables phénomènes d'endocytose, par exemple la phosphatase acide lysosomiale, dont le précurseur se trouve d'abord ancré à la membrane avec un large domaine extracytoplasmique avant d'être internalisé et dirigé vers les lysosomes, où l'extrémité carboxyterminale est clivée pour donner l'enzyme mûre [2]. Depuis 1990, plusieurs auteurs avaient suggéré que le signal nécessaire à l'internalisation, et probablement à la reconnaissance par les structures recouvertes, était un court peptide pouvant structuralement prendre une conformation en tour inversé. Au niveau du récepteur des LDL [3], cette séquence a pour for- mule Asn-Pro-Val-Tyr (NPVY en notation à une lettre) [4]. Au niveau de la phosphatase acide lysosomiale; un peptide Pro-Pro-Gly-Tyr semble jouer le même rôle [2]. Dans les deux cas, de courts peptides peuvent prendre, en effet, du fait de la présence de la tyrosine, une conformation en tour inversé. Toute mutation de la séquence NPVY du récepteur des LDL qui supprime cette conformation particulière, supprime également l'endocytose du récepteur [4]. Le mécanisme de l'endocytose de cette protéine, et probablement de la phosphatase acide, semble donc bien être la reconnaissance par des composants des puits et des vésicules recouverts d'une tyrosine exposée d'une manière particulière dans le contexte d'une structure en tour inversé.

[1. Pauloin A. médecine/sciences 1991 ; 7 : 561-8.]

[2. Eberle W, et al. Cell 1991; 67 : 1203-9.]

[3. Benlian P, Loux N. médecine/sciences 1991 ; 7 : 1052-61.]

[4. Bansal A, Gierasch LM. Cell $1991 ; 67$ : 1195-201.]

Localisation cellulaire du CFTR. Denning et al. (Howard Hughes Medical Institute, Iowa City, USA) [1] ont utilisé une technique d'immunofluorescence en microscopie confocale pour localiser la protéine CFTR (cystic fibrosis transmembrane conductance regulator) dans des lignées de cellules épithéliales sécrétrices, connues pour exprimer du CFTR endogène : T84, $\mathrm{CaCO}_{2}, \mathrm{HT} 29$ clone 19A. Des anticorps monoclonaux dirigés contre différentes régions de la protéine CFTR, le domaine $\mathrm{R}$ (régulateur), un segment $\mathrm{COOH}$-terminal (M1-4) et un domaine (M6-4) présumé extracellulaire ont été utilisés : les marquages témoins ont confirmé que ces trois anticorps précipitaient un polypeptide de 155-170 kDa dans des cellules $\mathrm{HeLa}$ et 3T3 exprimant un CFTR recombinant, mais ne marquaient pas des cellules dépourvues de CFTR - recombinant ou endogène. Dans les cellules épithéliales étudiées, le marquage apical pour le CFTR a été similaire à celui de 


\section{口RĖVES}

divers marqueurs connus de la membrane apicale, mais distinct du marquage des protéines membranaires baso-latérales; des coupes fines de monocouches cellulaires étaient également marquées au niveau de leur membrane apicale ; enfin, l'anticorps M6-4 du domaine extracellulaire marquait l'apex de cellules non perméabilisées aux ions. L'augmentation de la concentration intracellulaire en AMP cyclique ne modifiait pas le marquage CFTR apical, et stimulait la conductance au chlore, même à basse température, c'est-à-dire en l'absence d'insertion apicale de nouvelles vésicules intracellulaires ; ces données suggèrent que l'AMP cyclique stimule la perméabilité au chlore de la membrane apicale par activation directe de canaux existants plutôt qu'indirectement, par insertion dans la membrane de vésicules contenant du CFTR, c'est-à-dire probablement le canal chlore. Finalement, ces observations semblent démontrer que la protéine CFTR est bien localisée à la membrane apicale des trois variétés de cellules épithéliales étudiées, conclusion en accord avec celles, récemment publiées, faisant du CFTR le canal chlore de la membrane apicale.

[1. Denning GM, et al. J Clin Invest $1992 ; 89,339-49$.]

Le nez et le sexe : $y$ a-t-il des récepteurs olfactifs dans les testicules? Nous avons plusieurs fois présenté la méthode utilisée par l'équipe de G. Vassart, à l'Université de Bruxelles (Belgique), pour détecter des nouveaux membres de la grande famille des récepteurs liés aux protéines $\mathrm{G}\left([1]\right.$ et $m / s n^{\circ} 6$, vol. 7, p. 634]. C'est cette méthode qui a été utilisée pour caractériser des membres du groupe des récepteurs olfactifs $(\mathrm{m} / \mathrm{s}$ $n^{\circ} 6$, vol. 7, p. 616). Rappelons qu'il s'agit d'amplifier, par PCR, des fragments d'ADN à l'aide d'amorces reconnaissant des séquences très conservées dans les gènes codant pour ces récepteurs liés aux protéines $\mathrm{G}$. $\mathrm{M}$. Parmentier et al., du laboratoire de G. Vassart, viennent maintenant de démontrer que certains ADN complémentaires codant pour des récepteurs orphelins synthétisés dans le testicule correspondaient, en réalité, à des séquences de récepteurs olfactifs. Les gènes codant pour ces récepteurs sont extrêmement nombreux, probablement plusieurs centaines. Il se pourrait que tous ne soient pas exprimés dans la muqueuse olfactive, certains jouant peut-être un rôle physiologique lié au chimiotactisme dans d'autres cellules. Dans les testicules, le chimiotactisme est un phénomène évidemment important pour les spermatozoïdes, leur permettant de reconnaître et d'atteindre l'ovocyte à féconder. De fait, c'est dans la fraction de cellules testiculaires enrichie en cellules de la lignée germinale que Parmentier et al. détectent, par Northern blot, la plus grande quantité de messagers codant pour ces récepteurs. L'hétérogénéité de cette molécule semble grande, dans les testicules comme dans la muqueuse olfactive, puisque 20 espèces différentes ont été, à ce jour, caractérisées par l'équipe bruxelloise. Si les transcrits de récepteurs olf actifs sont effectivement traduits dans la lignée germinale, et les récepteurs correspondants présents sur les spermatozoïdes (ce qui, de l'avis même de l'équipe bruxelloise reste à démontrer), quelle est la signification de leur diversité ? Si l'hypothèse d'une attraction chimiotactique des gamètes mâles est correcte, pourquoi les ovocytes auraient-ils besoin d'émettre plusieurs types de signaux? Existe-t-il une sélectivité entre les signaux émis par des ovocytes individuels et l'attraction particulière de certains spermatozoïdes ? Une inadéquation entre les "phéromones" ovocytaires et les récepteurs testiculaires hypothétiques peut-elle expliquer certaines stérilités? On voit que cette notion, pure spéculation à ce jour, transposerait de manière surprenante le rôle bien connu des odeurs dans l'attraction sexuelle au déroulement de la danse nuptiale des spermatozoïdes autour des ovocytes. Une telle notion remettrait également en cause le caractère purement aléatoire de la fécondation d'un ovocyte donné par n'importe lequel des spermatozoïdes émis.

[1. Vassart $G$, et al. médecine/sciences $1990 ; 6: 985-90$.

[2. Parmentier M, et al. Nature 1992 ; $355: 453-5$. 